\title{
COMPOSITION SUM IDENTITIES RELATED TO THE DISTRIBUTION OF COORDINATE VALUES IN A DISCRETE SIMPLEX.
}

\author{
R. MILSON \\ DEPT. MATHEMATICS \& STATISTICS \\ DALHOUSIE UNIVERSITY \\ HALIFAX, N.S. B3H 3J5 \\ CANADA \\ MILSON@MATHSTAT.DAL.CA
}

Submitted: March 27, 2000; Accepted: April 13, 2000.

AMS Subject Classifications: 05A19, 05A20.

\begin{abstract}
Utilizing spectral residues of parameterized, recursively defined sequences, we develop a general method for generating identities of composition sums. Specific results are obtained by focusing on coefficient sequences of solutions of first and second order, ordinary, linear differential equations.

Regarding the first class, the corresponding identities amount to a proof of the exponential formula of labelled counting. The identities in the second class can be used to establish certain geometric properties of the simplex of bounded, ordered, integer tuples.

We present three theorems that support the conclusion that the inner dimensions of such an order simplex are, in a certain sense, more ample than the outer dimensions. As well, we give an algebraic proof of a bijection between two families of subsets in the order simplex, and inquire as to the possibility of establishing this bijection by combinatorial, rather than by algebraic methods.
\end{abstract}

\section{INTRODUCTION}

The present paper is a discussion of composition sum identities that may be obtained by utilizing spectral residues of parameterized, recursively defined sequences. Here we are using the term "composition sum" to refer to a sum whose index runs over all ordered lists of positive integers $p_{1}, p_{2}, \ldots, p_{l}$ that such that for a fixed $n$,

$$
p_{1}+\ldots+p_{l}=n .
$$

Spectral residues will be discussed in detail below.

Compositions sums are a useful device, and composition sum identities are frequently encountered in combinatorics. For example the Stirling numbers (of both kinds) have a

This research supported by a Dalhousie University grant. 
natural representation by means of such sums: [4, §51, §60]:

$$
s_{n}^{l}=\frac{n !}{l !} \sum_{p_{1}+\ldots+p_{l}=n} \frac{1}{p_{1} p_{2} \ldots p_{l}} ; \quad \mathfrak{S}_{n}^{l}=\frac{n !}{l !} \sum_{p_{1}+\ldots+p_{l}=n} \frac{1}{p_{1} ! p_{2} ! \ldots p_{l} !} .
$$

There are numerous other examples. In general, it is natural to use a composition sum to represent the value of quantities $f_{n}$ that depend in a linearly recursive manner on quantities $f_{1}, f_{2}, \ldots, f_{n-1}$. By way of illustration, let us mention that this point of view leads immediately to the interpretation of the $n^{\text {th }}$ Fibonacci number as the cardinality of the set of compositions of $n$ by $\{1,2\}[1,2.2 .23]$

To date, there are few systematic investigations of composition sum identities. The references known to the present author are [2] [3] [6]; all of these papers obtain their results through the use of generating functions. In this article we propose a new technique based on spectral residues, and apply this method to derive some results of an enumerative nature. Let us begin by describing one of these results, and then pass to a discussion of spectral residues.

Let $S^{3}(n)$ denote the discrete simplex of bounded, ordered triples of natural numbers:

$$
S^{3}(n)=\left\{(x, y, z) \in \mathbb{N}^{3}: 0 \leq x<y<z \leq n\right\} .
$$

In regard to this simplex, we may inquire as to what is more probable: a selection of points with distinct $y$ coordinates, or a selection of points with distinct $x$ coordinates. The answer is given by the following.

Theorem 1.1. For every cardinality $l$ between 2 and $n-1$, there are more l-element subsets of $S^{3}(n)$ with distinct $y$ coordinates, than there are l-element subsets with distinct $x$ coordinates.

Let us consider this result from the point of view of generating functions. The number of points with $y=j$ is $j(n-j)$. Hence the generating function for subsets with distinct $y$-values is

$$
Y(t)=\prod_{j=1}^{n-1}(1+j(n-j) t)
$$

where $t$ counts the selected points. The number of points with $x=n-j$ is $j(j-1) / 2$. Hence, the generating function for subsets with distinct $x$-values is

$$
X(t)=\prod_{j=2}^{n}\left(1+\frac{j(j-1)}{2} t\right) .
$$

The above theorem is equivalent to the assertion that the coefficients of $Y(t)$ are greater than the coefficients of $X(t)$. The challenge is to find a way to compare these coefficients.

We will see below this can be accomplished by re-expressing the coefficients in question as composition sums, and then employing a certain composition sum identity to make the comparison. We therefore begin by introducing a method for systematically generating such identities. 
THe EleCtronic Journal of COMbinatorics 7 (2000), \#R20

\section{The METHOD OF SPECTRAL RESIDUES}

Let us consider a sequence of quantities $f_{n}$, recursively defined by

$$
f_{0}=1, \quad(\nu-n) f_{n}=\sum_{j=0}^{n-1} a_{j n} f_{j}, \quad n=1,2, \ldots
$$

where the $a_{j k}, 0 \leq j<k$ is a given array of constants, and $\nu$ is a parameter. The presence of the parameter has some interesting consequences.

For instance, it is evident that if $\nu$ is a natural number, then there is a possibility that the relations (2.1) will not admit a solution. To deal with this complication we introduce the quantities

$$
\rho_{n}=\operatorname{Res}\left(f_{n}(\nu), \nu=n\right)
$$

and henceforth refer to them as spectral residues. The list $\rho_{1}, \rho_{2}, \ldots$ will be called the spectral residue sequence.

Proposition 2.1. If $\nu=n$ then the relations (2.1) do not admit a solution if $\rho_{n} \neq 0$, and admit multiple solutions if $\rho_{n}=0$.

Proof. If $\nu=n$, the relations in question admit a solution if and only if

$$
\left.\sum_{j=1}^{n-1} a_{j n} f_{j}\right|_{\nu=n}=0
$$

The left-hand side of the above equation is precisely, $\rho_{n}$, the $n^{\text {th }}$ spectral residue. It follows that if $\rho_{n}=0$, then the value of $f_{n}$ can be freely chosen, and that the solutions are uniquely determined by this value.

The above proposition is meant to indicate how spectral residues arise naturally in the context of parameterized, recursively defined sequences. However, our interest in spectral residues is motivated by the fact that they can be expressed as composition sums. To that end, let $\mathbf{p}=\left(p_{1}, \ldots, p_{l}\right)$ be an ordered list of natural numbers. We let

$$
s_{j}=p_{1}+\ldots+p_{j}, \quad j=1, \ldots, l
$$

denote the $j^{\text {th }}$ left partial sum and set

$$
|\mathbf{p}|=s_{l}=p_{1}+\ldots+p_{l} .
$$

Let us also define the following abbreviations:

$$
s_{\mathbf{p}}=\prod_{j=1}^{l-1} s_{j}, \quad a_{\mathbf{p}}=\prod_{j=1}^{l-1} a_{s_{j} s_{j+1}}
$$

Proposition 2.2.

$$
\rho_{n}=\sum_{|\mathbf{p}|=n} a_{\mathbf{p}} / s_{\mathbf{p}}
$$


Composition sum identities arise in this setting because spectral residue sequences enjoy a certain invariance property.

Let $\mathbf{f}=\left(f_{1}, f_{2}, \ldots\right)$ and $\mathbf{g}=\left(g_{1}, g_{2}, \ldots\right)$ be sequences defined, respectively by relation (2.1) and by

$$
g_{0}=1, \quad(\nu-n) g_{n}=\sum_{j=0}^{n-1} b_{j n} g_{j}, \quad n=1,2, \ldots
$$

Definition 2.3. We will say that $\mathbf{f}$ and $\mathbf{g}$ are unipotently equivalent if $g_{n}=f_{n}$ plus a $\nu$ independent linear combination of $f_{1}, \ldots, f_{n-1}$.

The motivation for this terminology is as follows. It is natural to represent the coefficients $a_{i j}$ and $b_{i j}$ by infinite, lower nilpotent matrices, call them $A$ and $B$. Let $D_{\nu}$ denote the diagonal matrix with entry $\nu-n$ in position $n+1$. The sequences $\mathbf{f}$ and $\mathbf{g}$ are then nothing but generators of the kernels of $D_{\nu}-A$ and $D_{\nu}-B$, respectively. The condition that $\mathbf{f}$ and $\mathrm{g}$ are unipotently equivalent amounts to the condition that $D_{\nu}-A$ and $D_{\nu}-B$ are related by a unipotent matrix factor.

Unipotent equivalence is, evidently, an equivalence relation on the set of sequences of type (2.1).

Proposition 2.4. The spectral residue sequence is an invariant of the corresponding equivalence classes.

Proof. The recursive nature of the $f_{k}$ ensures that $\operatorname{Res}\left(f_{k} ; \nu=n\right)$ vanishes for all $k<n$. The proposition now follows by inspection of Definition 2.3 .

The application of this result to composition identities is immediate.

Corollary 2.5. If $a_{i j}$ and $b_{i j}$ are nilpotent arrays of constants such that the corresponding $\mathbf{f}$ and $\mathbf{g}$ are unipotently equivalent, then necessarily

$$
\sum_{|\mathbf{p}|=n} a_{\mathbf{p}} / s_{\mathbf{p}}=\sum_{|\mathbf{p}|=n} b_{\mathbf{p}} / s_{\mathbf{p}}
$$

Due to its general nature, the above result does not, by itself, lead to interesting composition sum identities. In the search for useful applications we will limit our attention to recursively defined sequences arising from series solutions of linear differential equations. Consideration of both first and second order equations in one independent variable will prove fruitful. Indeed, in the next section we will show that the first-order case naturally leads to the exponential formula of labelled counting $[7, \S 3]$. The second-order case will be considered after that; it leads naturally to the type of result discussed in the introduction. 
THE Electronic Journal of combinatorics 7 (2000), \#R20

\section{Spectral Residues of FiRSt-ORDER EQUATIONS.}

Let $U=U_{1} z+U_{2} z^{2}+\ldots$ be a formal power series with zero constant term, and let $\phi(z)$ be the series solution of the following parameterized, first-order, differential equation:

$$
z \phi^{\prime}(z)+[U(z)-\nu] \phi(z)+\nu=0, \quad \phi(0)=1 .
$$

Equivalently, the coefficients of $\phi(z)$ must satisfy

$$
\phi_{0}=1, \quad(\nu-n) \phi_{n}=\sum_{j=0}^{n-1} U_{n-j} \phi_{j} .
$$

In order to obtain a composition sum identity we seek a related equation whose solution will be unipotently related to $\phi(z)$. It is well known that a linear, first-order differential equation can be integrated by means of a gauge transformation. Indeed, setting

$$
\begin{aligned}
& \sigma(z)=\sum_{k=1}^{\infty} U_{k} \frac{z^{k}}{k}, \\
& \psi(z)=\exp (\sigma(z)) \phi(z)
\end{aligned}
$$

our differential equation is transformed into

$$
z \psi^{\prime}(z)-\nu \psi(z)+\nu \exp (\sigma(z))=0 .
$$

Evidently, the coefficients of $\phi$ and $\psi$ are unipotently related, and hence we obtain the following composition sum identity.

Proposition 3.1. Setting $U_{\mathbf{p}}=\prod_{i} U_{p_{i}}$ for $\mathbf{p}=\left(p_{1}, \ldots, p_{l}\right)$ we have

$$
\sum_{n} \sum_{|\mathbf{p}|=n} \frac{U_{\mathbf{p}}}{s_{\mathbf{p}}} \frac{z^{n}}{n}=\exp \left(\sum_{k} U_{k} \frac{z^{k}}{k}\right) .
$$

The above identity has an interesting interpretation in the context of labelled counting, e.g. the enumeration of labelled graphs. In our discussion we will adopt the terminology introduced in H. Wilf's book [7]. For each natural number $k \geq 1$ let $\mathcal{D}_{k}$ be a set - we will call it a deck - whose elements we will refer to as pictures of weight $k$. A card of weight $k$ is a pair consisting of a picture of weight $k$ and a $k$-element subset of $\mathbb{N}$ that we will call the label set of the card. A hand of weight $n$ and size $l$ is a set of $l$ cards whose weights add up to $n$ and whose label sets form a partition of $\{1,2, \ldots, n\}$ into $l$ disjoint groups. The goal of labelled counting is to establish a relation between the cardinality of the sets of hands and the cardinality of the decks.

For example, when dealing with labelled graphs, $\mathcal{D}_{k}$ is the set of all connected $k$-graphs whose vertices are labelled by $1,2, \ldots, k$. A card of weight $k$ is a connected $k$-graph labelled by any $k$ natural numbers. Equivalently, a card can be specified as a picture and a set of natural number labels. To construct the card we label vertex 1 in the picture by the smallest label, vertex 2 by the next smallest label, etc. Finally, a hand of weight $n$ is an $n$-graph (not necessarily connected) whose vertices are labelled by $1,2, \ldots, n$. 
THE EleCtronic Journal of COMBinatorics 7 (2000), \#R20

Let $d_{k}$ denote the cardinality of $\mathcal{D}_{k}$ and set

$$
d(z)=\sum_{k} d_{k} \frac{z^{k}}{k !}
$$

Similarly let $h_{n l}$ denote the cardinality of the set of hands of weight $n$ and size $l$, and set

$$
h(y, z)=\sum_{n l} h_{n l} y^{l} \frac{z^{n}}{n !} .
$$

The exponential formula of labelled counting is an identity that relates the above generating functions. Here it is:

$$
h(y, z)=\exp (y d(z)) .
$$

To establish the equivalence of (3.2) and (3.3) we need to introduce some extra terminology. Consider a list of $l$ cards with weights $p_{1}, \ldots, p_{l}$ and label sets $S_{1}, \ldots, S_{l}$. We will say that such a list forms an ordered hand if

$$
\min \left(S_{i}\right)<\min \left(S_{i+1}\right), \quad \text { for all } i=1, \ldots, l-1 .
$$

Evidently, each hand (a set of cards) corresponds to a unique ordered hand (an ordered list of the same cards), and hence we seek a way to enumerate the set of all ordered hands of weight $n$ and size $l$.

Let us fix a composition $\mathbf{p}=\left(p_{1}, \ldots, p_{l}\right)$ of a natural number $n$, and consider a permutation $\pi=\left(\pi_{1}, \ldots, \pi_{n}\right)$ of $\{1, \ldots, n\}$. Let us sort $\pi$ according to the following scheme. Exchange $\pi_{1}$ and 1 and then sort $\pi_{2}, \ldots, \pi_{p_{1}}$ into ascending order. Next exchange $\pi_{p_{1}+1}$ and the minimum of $\pi_{p_{1}+1}, \ldots, \pi_{n}$ and then sort $\pi_{p_{1}+2}, \ldots, \pi_{p_{2}}$ into ascending order. Continue in an analogous fashion $l-2$ more times. The resulting permutation will describe a division of $\{1, \ldots, n\}$ into $l$ ordered blocks, with the blocks themselves being ordered according to their smallest elements. Call such a permutation p-ordered. Evidently, each p-ordered permutation can be obtained by sorting

$$
s_{\mathbf{p}} \times n \times \prod_{i}\left(p_{i}-1\right) !
$$

different permutations.

Next, let us note that an ordered hand can be specified in terms of the following ingredients: a composition $\mathbf{p}$ of $n$, one of $\prod_{i} d_{p_{i}}$ choices of pictures of weights $p_{1}, \ldots, p_{l}$, and a $\mathbf{p}$-ordered permutation. It follows that

$$
h_{n l}=\sum_{\substack{|\mathbf{p}|=n \\ \mathbf{p}=\left(p_{1}, \ldots, p_{l}\right)}} \frac{n !}{s_{\mathbf{p}} \times n \times \prod_{i}\left(p_{i}-1\right) !} \prod_{i} d_{p_{i}} .
$$

Finally, we can establish the equivalence of (3.2) and (3.3) by setting

$$
U_{k}=\frac{d_{k}}{(k-1) !} y
$$


THE Electronic Journal of combinatorics 7 (2000), \#R20

\section{Spectral Residues of SECOND-ORDER EQUATIONS.}

Let $U=U_{1} z+U_{2} z^{2}+\ldots$ be a formal power series with zero constant term, and let $\phi(z)$ be the series solution of the following second-order, linear differential equation:

$$
z^{2} \phi^{\prime \prime}(z)+(1-\nu) z \phi^{\prime} z+U(z) \phi(z)=0, \quad \phi(0)=1
$$

Equivalently, the coefficients of $\phi(z)$ are determined by

$$
\phi_{0}=1, \quad n(\nu-n) \phi_{n}=\sum_{j=0}^{n-1} U_{n-j} \phi_{j} .
$$

Two remarks are in order at this point. First, the class of equations described by (4.4) is closely related to the class of self-adjoint second-order equations. Indeed, conjugation by a gauge factor $z^{\nu / 2}$ transforms (4.4) into self-adjoint form with potential $U(z)$ and energy $\nu^{2} / 4$. The solutions of the self-adjoint form are formal series multiplied by $z^{\nu / 2}$, so nothing is lost by working with the "nearly" self-adjoint form (4.4).

Second, there is no loss of generality in restricting our focus to the self-adjoint equations. Every second-order linear equation can be gauge-transformed into self-adjoint form, and as we saw above, spectral residue sequences are invariant with respect to gauge transformations. Indeed, as we shall demonstrate shortly, the potential $U(z)$ is uniquely determined by its corresponding residue sequence.

Proposition 4.1. The spectral residues corresponding to (4.4) are

$$
\rho_{n}=\frac{1}{n} \sum_{|\mathbf{p}|=n} \frac{U_{\mathbf{p}}}{s_{\mathbf{p}} s_{\mathbf{p}^{\prime}}},
$$

where as before, for $\mathbf{p}=\left(p_{1}, \ldots, p_{l}\right)$, we write $U_{\mathbf{p}}$ for $\prod_{i} U_{p_{i}}$, and write $\mathbf{p}^{\prime}$ for the reversed composition $\left(p_{l}, p_{l-1}, \ldots, p_{1}\right)$.

Since $\rho_{n}=U_{n} / n$ plus a polynomial of $U_{1}, \ldots, U_{n-1}$, it is evident that the spectral residue sequence completely determines the potential $U(z)$. An explicit formula for the inverse relation is given in [5].

Interesting composition sum identities will appear in the present context when we consider exactly-solvable differential equations. We present three such examples below, and discuss the enumerative interpretations in the next section. In each case the exact solvability comes about because the equation is gauge-equivalent to either the hypergeometric, or the confluent hypergeometric equation. Let us also remark - see [5] for the details - that these equations occupy an important place within the canon of classical quantum mechanics, where they correspond to various well-known exactly solvable one-dimensional models.

\section{Proposition 4.2.}

$$
\sum_{\substack{\mathbf{p}=\left(p_{1}, \ldots, p_{l}\right) \\|\mathbf{p}|=n}} \frac{(n-1) !}{s_{\mathbf{p}}} \frac{(n-1) !}{s_{\mathbf{p}^{\prime}}}\left(\prod_{i} p_{i}\right) t^{l}=\prod_{j=1}^{n}\{t+j(j-1)\}
$$


THE Electronic Journal of combinatorics 7 (2000), \#R20

Proof. By Proposition 4.1, the left hand side of the above identity is $n !(n-1)$ ! times the $n^{\text {th }}$ spectral residue corresponding to the potential

$$
U(z)=\frac{t z}{(z-1)^{2}}=t \sum_{k} k z^{k} .
$$

Setting

$$
t=\alpha(1-\alpha)
$$

and making a change of gauge

$$
\phi(z)=(1-z)^{\alpha} \psi(z)
$$

transforms (4.4) into

$$
z^{2} \psi^{\prime \prime}(z)+(1-\nu) \psi^{\prime}(z)-\frac{z}{1-z}\left\{2 \alpha z \psi^{\prime}(z)+\alpha(\alpha-\nu) \psi(z)\right\}=0 .
$$

Multiplying through by $(1-z) / z$ and setting

$$
\gamma=1-\nu, \quad \beta=\alpha-\nu
$$

we recover the usual hypergeometric equation

$$
z(1-z) \psi^{\prime \prime}(z)+\{\gamma+(1-\alpha-\beta) z\} \psi^{\prime}(z)-\alpha \beta \psi(z)=0 .
$$

It follows that

$$
\psi_{n}=\frac{(\alpha)_{n}(\alpha-\nu)_{n}}{n !(1-\nu)_{n}}
$$

and hence the $n^{\text {th }}$ spectral residue is given by

$$
\rho_{n}=(-1)^{n} \frac{\prod_{j=1}^{n}(\alpha-j)(\alpha+j-1)}{n !(n-1) !},
$$

or equivalently by

$$
\rho_{n}=\frac{\prod_{j=1}^{n}(t+j(j-1))}{n !(n-1) !} .
$$

The asserted identity now follows from the fundamental invariance property of spectral residues.

\section{Proposition 4.3.}

$$
\sum_{\substack{\mathbf{p}=\left(p_{1}, \ldots, p_{l}\right) \\ p_{i} \in\{1,2\} \\|\mathbf{p}|=n}} \frac{(n-1) !}{s_{\mathbf{p}}} \frac{(n-1) !}{s_{\mathbf{p}^{\prime}}} t^{n-l}=\prod_{k}\left(1+k^{2} t\right),
$$

where the right hand index $k$ varies over all positive integers $n-1, n-3, n-5, \ldots$. 
THE EleCtronic Journal of COMBinatorics 7 (2000), \#R20

Proof. As in the preceding proof, Proposition 4.1 shows that the left hand side of the present identity is $n !(n-1)$ ! times the $n^{\text {th }}$ spectral residue corresponding to the potential

$$
U(z)=z+t z^{2}
$$

Setting

$$
t=-\omega^{2}
$$

and making a change of gauge

$$
\phi(z)=\exp (\omega z) \psi(z)
$$

transforms (4.4) into

$$
z^{2} \psi^{\prime \prime}(z)+(1-\nu) z \psi^{\prime}(z)+2 \omega z^{2} \psi^{\prime}(z)+z(\omega(1-\nu)+1) \psi(z)=0 .
$$

Dividing through by $z$ and setting

$$
\gamma=1-\nu, \quad 1=\omega(2 \alpha+\nu-1)
$$

we obtain the following scaled variation of the confluent hypergeometric equation:

$$
z \psi^{\prime \prime}(z)+(\gamma+2 \omega z) \psi^{\prime}(z)+2 \omega \alpha \psi(z)=0 .
$$

It follows that

$$
\psi_{n}=\frac{(-2 \omega)^{2}(\alpha)_{n}}{n !(\gamma)_{n}}
$$

and hence that

$$
\begin{aligned}
\rho_{n} & =\frac{\prod_{k=0}^{n-1}(1+\omega(2 k+1-n))}{n !(n-1) !} \\
& =\frac{\prod_{k=0}^{\left\lfloor\frac{n-1}{2}\right\rfloor}\left(1+t(n-1-2 k)^{2}\right)}{n !(n-1) !} .
\end{aligned}
$$

The asserted identity now follows from the fundamental invariance property of spectral residues.

Proposition 4.4.

$$
\sum_{\substack{\mathbf{p}=\left(p_{1}, \ldots, p_{l}\right) \\ p_{i} \text { odd } \\|\mathbf{p}|=n}} \frac{(n-1) !}{s_{\mathbf{p}}} \frac{(n-1) !}{s_{\mathbf{p}^{\prime}}}\left(\prod_{i} p_{i}\right) t^{\frac{n-l}{2}}=\prod_{k}\left\{1+\left(k^{4}-k^{2}\right) t\right\},
$$

where the right hand index $k$ ranges over all positive integers $n-1, n-3, n-5, \ldots$

Proof. By Proposition 4.1, the left hand side of the present identity is $n !(n-1) ! t^{n / 2}$ times the $n^{\text {th }}$ spectral residue corresponding to the potential

$$
U(z)=\frac{1}{2 \sqrt{t}}\left(\frac{z}{(1-z)^{2}}+\frac{z}{(1+z)^{2}}\right)=\frac{1}{\sqrt{t}} \sum_{k \text { odd }} k z^{k} .
$$


The rest of the proof is similar to, but somewhat more involved than the proofs of the preceding two Propositions. Suffice it to say that with the above potential, equation (4.4) can be integrated by means of a hypergeometric function. This fact, in turn, serves to establish the identity in question. The details of this argument are to be found in [5].

\section{Distribution of COORdinate VAlues in A Discrete Simplex}

In this section we consider enumerative interpretations of the composition sum identities derived in Proposition 4.2, 4.3, 4.4. Let us begin with some general remarks about compositions.

There is a natural bijective correspondence between the set of compositions of $n$ and the powerset of $\{1, \ldots, n-1\}$. The correspondence works by mapping a composition $\mathbf{p}=$ $\left(p_{1}, \ldots, p_{l}\right)$ to the set of left partial sums $\left\{s_{1}, \ldots, s_{l-1}\right\}$, henceforth to be denoted by $L_{\mathbf{p}}$. It may be useful to visualize this correspondence it terms of a "walk" from 0 to $n$ : the composition specifies a sequence of displacements, and $L_{\mathbf{p}}$ is the set of points visited along the way. One final item of terminology: we will call two compositions $\mathbf{p}$, $\mathbf{q}$ of $n$ complimentary, whenever $L_{\mathbf{p}}$ and $L_{\mathbf{q}}$ disjointly partition $\{1, \ldots, n-1\}$.

Now let us turn to the proof of Theorem 1.1. As was mentioned in the introduction, this Theorem is equivalent to the assertion that the coefficients of

$$
Y(t)=\prod_{j=1}^{n-1}(1+j(n-j) t)
$$

are greater than the corresponding coefficients of

$$
X(t)=\prod_{j=2}^{n}\left(1+\frac{j(j-1)}{2} t\right) .
$$

Rewriting the former function as a composition sum we have

$$
Y(t)=\sum_{\substack{\mathbf{p}=\left(p_{1}, \ldots, p_{l}\right) \\|\mathbf{p}|=n}} s_{\mathbf{p}} s_{\mathbf{p}^{\prime}} t^{l},
$$

or equivalently

$$
Y(t)=\sum_{\substack{\mathbf{p}=\left(p_{1}, \ldots, p_{l}\right) \\|\mathbf{p}|=n}} \frac{(n-1) !}{s_{\mathbf{p}}} \frac{(n-1) !}{s_{\mathbf{p}^{\prime}}} t^{n-l} .
$$

On the other hand, Proposition 4.2 allows us to write

$$
X(t)=\sum_{\substack{\mathbf{p}=\left(p_{1}, \ldots, p_{l}\right) \\|\mathbf{p}|=n}} \frac{(n-1) !}{s_{\mathbf{p}}} \frac{(n-1) !}{s_{\mathbf{p}^{\prime}}}\left(\prod_{i} \frac{p_{i}}{2^{p_{i}-1}}\right) t^{n-l} .
$$


THe EleCtronic Journal of COMbinatorics 7 (2000), \#R20

It now becomes a straightforward matter to compare the coefficients of $Y(t)$ to those of $X(t)$. Indeed the desired conclusion follows from the rather obvious inequality:

$$
k \leq 2^{k-1}, \quad k=1,2,3 \ldots,
$$

the inequality being strict for $k \geq 3$.

Let us now turn to an enumerative interpretation of the composition sum identity featured in Proposition 4.3. In order to state the upcoming result we need to define two notions of sparseness for subsets of $S^{3}(n)$. Let us call a multiset $M$ of integers sparse if $M$ does not contain duplicates, and if

$$
|a-b| \geq 2
$$

for all distinct $a, b \in M$. Let us also say that a multiset $M$ is 2-sparse if $M$ does not contain duplicates, and if there do not exist distinct $a, b \in M$ such that

$$
\lfloor a / 2\rfloor=\lfloor b / 2\rfloor .
$$

It isn't hard to see that sparseness is a more restrictive notion than 2-sparseness, i.e. if $M$ is sparse, then it is necessarily 2-sparse, but not the other way around. For example, the set

$$
\{1,3,4,7\}
$$

is not sparse, but it is 2-sparse.

We require one other item of notation. For $A \subset S^{3}(n)$ we let $\pi_{x}(A)$ denote the multiset of $x$-coordinates of points in $A$, and let $\pi_{y}(A)$ denote the multiset of $y$-coordinates. We are now ready to state

Theorem 5.1. For every cardinality $l$ between 2 and $n-1$, there are more l-element subsets $A$ of $S^{3}(n)$ such that $\pi_{y}(A)$ is sparse, than there are l-element subsets $A$ such that $\pi_{x}(A)$ is sparse. Indeed, the number of l-element subsets $A$ of $S^{3}(n)$ such that $\pi_{y}(A)$ is sparse is equal to the number of l-element subsets $A$ of $S^{3}(n)$ such that $\pi_{x}(A)$ is merely 2-sparse.

Proof. Let $\mathbf{p}$ be a composition of $n$. Let us begin by noting that the corresponding $L_{\mathbf{p}}$ is sparse if and only if the complimentary composition consists of 1's and 2's only. It therefore follows that the enumerating function for $A \subset S^{3}(n)$ such that $\pi_{y}(A)$ is sparse is

$$
\sum_{\substack{\mathbf{p}=\left(p_{1}, \ldots, p_{l}\right) \\ p_{i} \in\{1,2\} \\|\mathbf{p}|=n}} \frac{(n-1) !}{s_{\mathbf{p}}} \frac{(n-1) !}{s_{\mathbf{p}^{\prime}}} t^{n-l} .
$$

On the other hand the number of $(x, y, z) \in S^{3}(n)$ such that $x \in\{2 k, 2 k+1\}$ for any given $k$ is precisely

$$
\left(\begin{array}{c}
n-2 k \\
2
\end{array}\right)+\left(\begin{array}{c}
n-2 k-1 \\
2
\end{array}\right)=(n-2 k-1)^{2} .
$$


Hence the enumerating function for $A \subset S^{3}(n)$ such that $\pi_{x}(A)$ is 2-sparse is

$$
\prod_{k=0}^{\lfloor(n-1) / 2\rfloor}\left(1+(n-2 k-1)^{2} t\right) \text {. }
$$

The two enumerating functions are equal by Proposition 4.3.

Finally, let us consider an enumerative interpretation of the composition sum identity featured in Proposition 4.3. The setting for this result will be $S^{5}(n)$, the discrete simplex of all bounded, ordered 5 -tuples $\left(x_{1}, x_{2}, x_{3}, x_{4}, x_{5}\right)$. For $A \subset S^{5}(n)$ we will use $\pi_{i}(A), i=1, \ldots, 5$ to denote the corresponding multiset of $x_{i}$ coordinate values.

Theorem 5.2. For every cardinality $l$ between 2 and $n-3$, there are more l-element subsets $A$ of $S^{5}(n)$ such that $\pi_{3}(A)$ is sparse, than there are l-element subsets $A$ such that $\pi_{1}(A)$ is 2-sparse.

Proof. Let us note that the number of points in $S^{5}(n)$ such that $x_{3}=j+1$ is given by

$$
\left(\begin{array}{c}
j+1 \\
2
\end{array}\right)\left(\begin{array}{c}
n-j-1 \\
2
\end{array}\right) \text {. }
$$

Hence, the enumerating function for the first class of subsets is given by

$$
X_{3}(t)=\sum_{\substack{\mathbf{p}=\left(p_{1}, \ldots, p_{l}\right) \\ p_{i} \in\{1,2\} \\|\mathbf{p}|=n-2}}\left\{\prod_{j \notin L_{\mathbf{p}}} \frac{j(j+1)(n-j-1)(n-j-2)}{4}\right\} t^{n-2-l} .
$$

Now there is a natural bijection between the set of compositions of $n-2$ by $\{1,2\}$ and the set of compositions of $n-1$ by odd numbers. The bijection works by prepending a 1 to a composition of the former type, and then performing substitutions of the form

$$
(\ldots, k, 2, \ldots) \mapsto(\ldots, k+2, \ldots), \quad k \text { odd }
$$

Consequently, we can write

$$
X_{3}(t)=\sum_{\substack{\mathbf{p}=\left(p_{1}, \ldots, p_{l}\right) \\ p_{i} \text { odd } \\|\mathbf{p}|=n-1}} \frac{n !}{s_{\mathbf{p}}} \frac{n !}{s_{\mathbf{p}^{\prime}}}\left(\frac{t}{4}\right)^{(n-1-l) / 2} .
$$

Turning to the other class of subsets, the number of points $\left(x_{1}, \ldots, x_{5}\right)$ that satisfy

$$
x_{1} \in\{2 j, 2 j+1\}
$$

is given by

$$
\left(\begin{array}{c}
n-2 j \\
4
\end{array}\right)+\left(\begin{array}{c}
n-2 j-1 \\
4
\end{array}\right)=\frac{(n-2 j-2)^{4}-(n-2 j-2)^{2}}{12}
$$


Consequently the enumerating function for subsets $A$ such that $\pi_{1}(A)$ is 2-sparse is given by

$$
X_{1}(t)=\prod_{k}\left(1+\left(k^{4}-k^{2}\right) \frac{t}{12}\right),
$$

where $k$ ranges over all positive integers $n-2, n-4, \ldots$ Next, using the identity in Proposition 4.4 we have

$$
X_{1}(t)=\sum_{\substack{\mathbf{p}=\left(p_{1}, \ldots, p_{l}\right) \\ p_{i} \text { odd } \\|\mathbf{p}|=n-1}} \frac{(n-1) !}{s_{\mathbf{p}}} \frac{(n-1) !}{s_{\mathbf{p}^{\prime}}}\left(\prod_{i} \frac{p_{i}}{3^{\left(p_{i}-1\right) / 2}}\right)\left(\frac{t}{4}\right)^{(n-1-l) / 2} .
$$

Using (5.5) it now becomes a straightforward matter to compare $X_{1}(t)$ to $X_{3}(t)$. Indeed, the desired conclusion follows from the following evident inequality:

$$
k \leq 3^{(k-1) / 2}, \quad k=1,3,5, \ldots,
$$

the inequality being strict for $k \geq 5$.

\section{Conclusion}

The above discussion centers around two major themes: spectral residues, and the distribution of coordinate values in a simplex of bounded, ordered integer tuples. In the first case, we have demonstrated that the method of spectral residues leads to composition sum identities with interesting interpretations. We have considered here parameterized recursive relations corresponding to first and second-order linear differential equations in one independent variable. The next step in this line of inquiry would be to consider other classes of parameterized recursive relations - perhaps non-linear, perhaps corresponding to partial differential equations - in the hope that new and useful composition sum identities would follow.

In the second case, we have uncovered an interesting geometrical property of the order simplex. Theorems 1.1, 5.1, 5.2 support the conclusion that the middle dimensions of an order simplex are more "ample" then the outer dimensions. However the 3 results we have been able to establish all depend on very specific identities, and do not provide a general tool for the investigation of this phenomenon. To put it another way, our results suggest the following

Conjecture 6.1. Let $N$ be a natural number greater than 2 and $d$ a natural number strictly less than $N / 2-1$. Let $n \geq N$ be another natural number. For every sufficiently small cardinality $l$, there are more l-element subsets of $S^{N}(n)$ with distinct $x_{d+1}$ coordinates, than there are l-element subsets with distinct $x_{d}$ coordinates.

It would also be interesting to see whether this conjecture holds if we consider subsets of points with sparse, rather than distinct sets of coordinate values. 
THE Electronic Journal of combinatorics 7 (2000), \#R20

Finally, Theorem (5.1) deserves closer scrutiny, because it describes a bijection of sets, rather than a mere comparison. It is tempting to conjecture that this bijection has an enumerative explanation based on some combinatorial algorithm.

\section{REFERENCES}

[1] Goulden, I. and Jackson, D., Combinatorial Enumeration, Wiley, New York, 1983.

[2] V. E. Hoggat Jr., D. A. Lind, Compositions and Fibonacci numbers, Fibonacci Quarterly, 7 (1969), $253-266$.

[3] N. P. Homenko, V. V. Strok, Certain combinatorial identities for sums of composition coefficients. Ukrain. Mat. Zh. 23 (1971), 830-837.

[4] Jordan, C., Calculus of Finite Differences, Chelsea, New York, 1947.

[5] Milson, R., Spectral residues of second-order differential equations: a new methodology for summation identities and inversion formulas, preprint math-ph/9912007.

[6] Moser, L. and Whitney, E., Weighted Compositions, Can. Math. Bull., 4:39-43 (1961).

[7] Wilf, H., "generatingfunctionology", Academic Press, 1990. 